\title{
Multi-resonance orbital model of HF QPOs
}

\author{
Zdeněk Stuchlík, Andrea Kotrlová and Gabriel Török \\ Institute of Physics, Faculty of Philosophy and Science, Silesian University in Opava, \\ Bezručovo nám. 13, CZ-74601 Opava, Czech Republic \\ email: zdenek.stuchlik@fpf.slu.cz, andrea.kotrlova@fpf.slu.cz
}

\begin{abstract}
Using known frequencies of the twin peak high-frequency quasiperiodic oscillations (HF QPOs) and known mass $M$ of the central black hole, the black-hole dimensionless spin $a$ can be determined assuming a concrete version of the resonance model. However, large range of observationally limited values of the black hole mass implies a low precision of the spin estimates. We discuss the possibility of higher precision of the black hole spin $a$ measurements in the framework of multi-resonance model inspired by observations of more than two HF QPOs in some black hole sources. We determine the spin and mass dependence of the twin peak frequencies with a general rational ratio $n: m$ assuming a non-linear resonance of oscillations with the epicyclic and Keplerian frequencies or their combinations. In the multi-resonant model, the twin peak resonances are combined properly to give the observed frequency set. We focus on the special case of duplex frequencies, when the top, bottom, or mixed frequency is common at two different radii where the resonances occur giving triple frequency sets.
\end{abstract}

Keywords. accretion, accretion disks - X-rays: binaries - black hole physics

\section{Multi-resonance models with Keplerian and epicyclic oscillations}

The standard orbital resonance model assumes non-linear resonance between oscillation modes of an accretion disc orbiting a central object, here considered to be a rotating Kerr black hole. The frequency of the oscillations is related to the Keplerian frequency $\nu_{\mathrm{K}}$ (orbital frequency of tori), or the radial $\nu_{r}$ and vertical $\nu_{\theta}$ epicyclic frequencies of the circular test particle motion.

\subsection{More resonances sharing one specific radius}

This special case allows existence of so called strong resonant phenomena when two (or more) versions of resonance could occur at the same radius allowing cooperative effects between the resonances (Stuchlík et al. 2008). Of course, such a situation is allowed for black holes with a specific spin only. Of special interest seems to be the case of the "magic" spin $a=0.983$, when the Keplerian and epicyclic frequencies are in the ratio $\nu_{\mathrm{K}}: \nu_{\theta}: \nu_{r}=3: 2: 1$ at the common radius $x \equiv r / M=2.395$, see Fig. 1 .

\subsection{Resonances occurring at two specific radii - triple frequency sets}

In general, we can expect the oscillations to be excited at two different radii of the accretion disc and to enter the resonance in the framework of different versions of the resonance model (i.e., four frequency set is observed generally). In special cases, for some specific values of the black hole spin, two twin peak QPOs observed at the radii $x_{n: m}$ and $x_{n^{\prime}: m^{\prime}}$ have the top (see Fig. 1), bottom or mixed (the bottom at the inner radius and the top in the outer radius, or vice versa) frequencies identical (Stuchlík et al. 2012). Such situations can be characterized by sets of only three frequencies (upper $\nu_{\mathrm{U}}$, middle $\nu_{\mathrm{M}}$ and lower $\nu_{\mathrm{L}}$ ) with ratio $\nu_{\mathrm{U}}: \nu_{\mathrm{M}}: \nu_{\mathrm{L}}=s: t: u$.

Let us consider a simple situation with the "top identity" of the upper frequencies in two direct resonances between the radial $\nu_{r}$ and vertical $\nu_{\theta}$ epicyclic oscillations at two different radii $x_{p}, x_{p^{\prime}}$ with $p^{1 / 2}=m: n, p^{1 / 2}=m^{\prime}: n^{\prime}$. The condition 

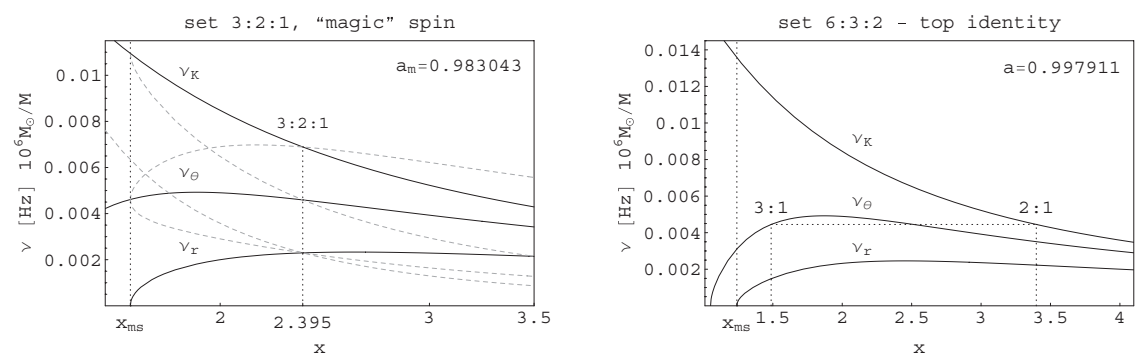

Figure 1. Left: The special case of a "magic" spin, when the strongest resonances could occur at the same radius. For completeness we present the relevant simple combinational frequencies $\nu_{\theta}-\nu_{r}, \nu_{\theta}+\nu_{r}, \nu_{\mathrm{K}}-\nu_{\theta}, \nu_{\mathrm{K}}-\nu_{r}$ (grey dashed lines). Right: The case of the duplex frequencies when the top frequency is common at two different radii where the resonances occur giving triple frequency ratio set.

$\nu_{\theta}\left(a, x_{p}\right)=\nu_{\theta}\left(a, x_{p^{\prime}}\right)$ is then transformed to the relation

$$
\alpha_{\theta}^{1 / 2}\left(a, x_{p}\right)\left(x_{p}^{3 / 2}+a\right)^{-1}=\alpha_{\theta}^{1 / 2}\left(a, x_{p^{\prime}}\right)\left(x_{p^{\prime}}^{3 / 2}+a\right)^{-1}
$$

which uniquely determines the black hole spin $a$, since the radii $x_{p}$ and $x_{p^{\prime}}$ are related to the spin $a$ by the resonance conditions

$$
a=a^{\theta / r}(x, p) \equiv \frac{\sqrt{x}}{3(p+1)}\{2(p+2)-\sqrt{(1-p)[3 x(p+1)-2(2 p+1)]}\}
$$

for $a^{\theta / r}(x, p)$ and $a^{\theta / r}\left(x, p^{\prime}\right)$, respectively. When two different resonances are combined, we proceed in the same manner (Stuchlík et al. 2012). The black hole spin $a$ is given by the types of the two resonances and the ratios $p, p^{\prime}$, quite independently of the black hole mass $M$. A detailed table guide across all the possible triple frequency sets and related values of the black hole spin $a$ (limited by $n \leqslant 4$ ) is presented in Stuchlík et al. (2012).

\section{Conclusions}

The multi-resonance model of HF QPOs can be considered as a promising approach to understand the observational data from black holes sources. The special triple frequency set method determines the black hole spin precisely, but not uniquely, as in general the same frequency set could occur for different values of the spin within different versions of the resonance model. In such situations the black hole spin estimates coming from the spectra fitting and the line profile model could be relevant in determining the proper versions of the resonant model. When the black hole spin is found, its mass can be determined from the magnitude of the observed frequencies. The efficiency of the black hole spin determination by using the triple frequency set ratios grows strongly with growing precision of the frequency measurements. The prepared new space X-ray mission LOFT proposes sensibility of the observational instruments high enough to reach data that could be precise enough to make application of the triple frequency set method realistic.

\section{Acknowledgements}

The authors acknowledge the research grant GAČR 202/09/0772 and the project CZ.1.07/2.3.00/

20.0071 "Synergy" supporting international collaboration of the Institute of Physics at SU Opava.

\section{References}

Stuchlík, Z., Kotrlová, A., \& Török, G. 2008, AcA, 58, 441

Stuchlík, Z., Kotrlová, A., \& Török, G. 2012, A\&A, submitted 